\title{
Plant Disease Detection and Monitoring Using Artificial Neural Network
}

\author{
Stella I. Orakwue and Nkolika O. Nwazor \\ ${ }^{1,2}$ Electrical/Electronic Engineering Department, University of Port Harcourt, Nigeria.
}

\begin{abstract}
Fungi have been identified as a major threat to crop production in the world. In this study, methods of improving the performance of plant disease detection and prediction using artificial neural network techniques are presented. The hyperspectral fungi dataset of 21 plant species were collected and trained using back propagation algorithms of an artificial neural network to improve the conventional hyperspectral sensor. The system was modelled using self-defining equations and universal modelling diagrams and then implemented in the neural network toolbox in Matlab. The system was tested validated and the result showed a fungi detection accuracy of $96.61 \%$ and the percentage increment was $19.53 \%$.
\end{abstract}

Keywords: plant disease, artificial neural network, backpropagation algorithms

\section{Introduction}

Today, agriculture has been the major source of food production, especially since the incidence of COVID19 , which resulted in the ban of international and interstate borders restricting the importation of food items from other parts of the world. However, the successful cultivation and production of these crops are where the challenges lie due to their varieties ranging from food to cash crops and the constraint affecting various factors of productions like the cost of labour, land, capital among others. According to [1], growing crop makes a farmer be confident of himself because he has created a new way of life, which is a beautiful activity, however, in order to produce healthy and high yield, this process requires steady maintenance and various farm activities and as a result, has become a major challenge.

Recently some of these problems have been solved by employing various means like the application of fertilizers to boost crop production rate, loan assistance from the bank of agriculture to support the cost of labour, legislative ideas like operation feed the nation, pesticides to help optimize pest control performance and lots more. However, despite these efforts applied, it is still difficult to guarantee optimized crop performance due to certain factors of nature like harsh weather conditions, plant diseases, pests, viruses, bacteria, and toxic soil effects among others.

According to [2-4] accurate estimation of how plant disease affects both quantity and quality of agricultural products negatively, the extent of disease impact are important factors for breeding of plant, yielding of the crop, horticulture, improvement of the efficacy of fungicide as well as fundamental and applied plant research works. Today, this has limited and discouraged many farmers, hindering their performance and rate of crop production. The fear of pests has hindered huge investment in agriculture by most farmers, thus forcing them into subsistence form of agricultural practice. These and more factors have affected the rate of food production and the price of food has skyrocketed [5].

To solve these problems, lots of hopes have been focused on the research domain to propose a solution to help optimize crop performance and pest disease control. This has resulted in various hypotheses from some of the best minds in the research field, thus proposing techniques like the use of real-time monitoring robots, application of Internet of Things based supervisory control and data acquisition schemes, pest control techniques, precision agriculture, smart farming, manual monitoring approaches, estimation of visual observation of plant disease by human, evaluation of the morphological features in pathogen identification 
microscopically, molecular, use of microbiological techniques for diagnosis, serological [6] and lots more. But these methods despite the success achieved have certain constraints like high cost of implementations, inaccuracy, complexity in design, poor response to disease detection, false prediction rate and lots more.

Therefore, there is a need for improved simple user-friendly, cost effective and intelligent systems, which monitors the crop performance intelligently and predict disease in real time using any artificial intelligence technique.

\section{The Proposed System}

The proposed system is focused on improving the sensing performance of the characterized system and the quality of data collected and finally making the system intelligent. To achieve this, a spectral model of the sensing element is used and the output is processed using a spectral data processing model or algorithm. The processed data is feed-forward to the artificial intelligent technique, which was already trained with plant fungi dataset extracted from infected plants. The training processes classified the data trained and predict the output as either fungus or not, if fungi were detected, the data is stored in the temporal memory bank. After two days if fungi are still detected then the communication module is triggered to activate the alarm of the buzzer notification system.

\section{Design Methodology}

The methodology employed for the development of the proposed system is the neural network techniques, geometric approach and computer aided software engineering methodology. This will guide the system design as presented below;

\section{A. The Plant Model}

This model defined the relationship of how leaf chemistry, structure and orientation interact with the irradiance from the sun and other environmental factors. When a plant leaf intercepts light bean, it can be partly reflected, absorbed or transmitted. The total sum of the reflectance $\mathrm{R}$, transmittance $\mathrm{T}$ and absorbance $\mathrm{A}$ is equal to one when expressed in coefficient as shown using the equation 1 ;

$$
R+T+A=1
$$

From the model in equation 1 , the summation of the reflectance, transmittance and absorbance presented the plant model.

\section{B. The Artificial Intelligence technique}

Artificial neural network is the technique used here to improve the system performance. This system is trained using a fungi dataset to develop a reference fungi model, which will be used to classify the data processed before predicting the output as threat or no threat to the farm. The training data set is described as shown in figure 1. 


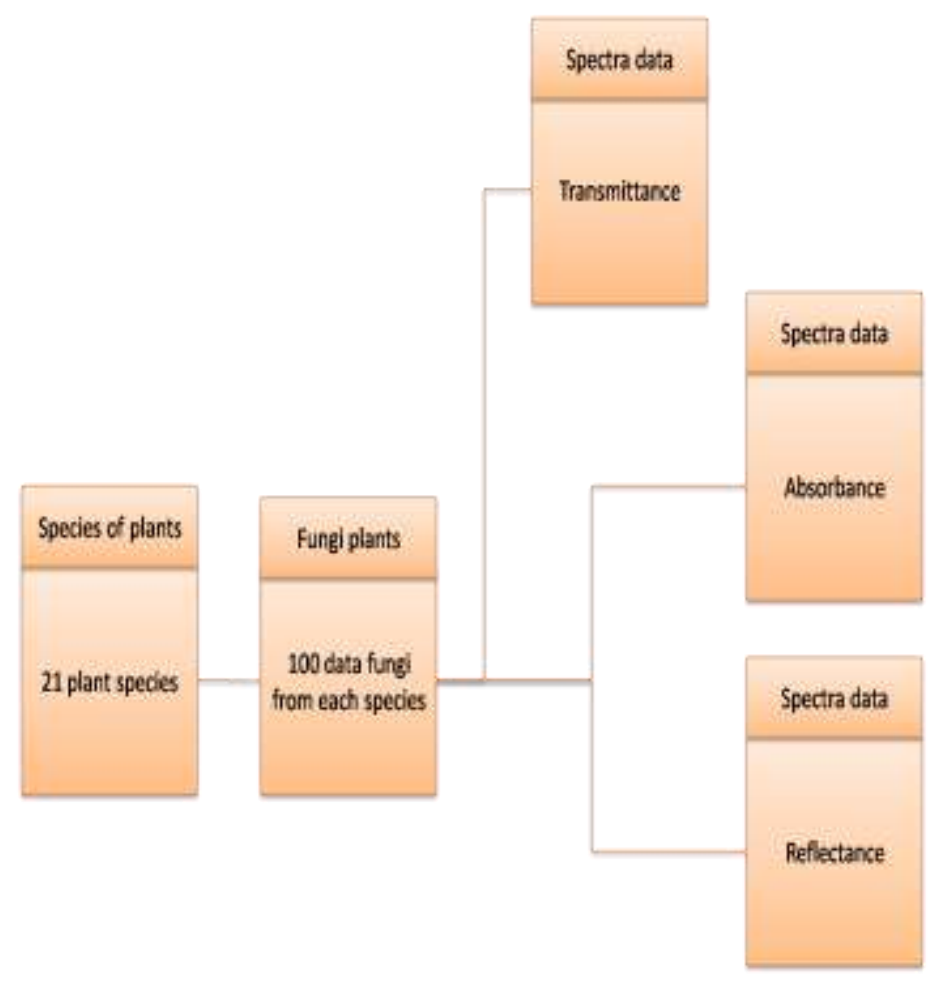

Fig. 1 Data Model

\section{The Data model of the fungi dataset}

The data model presented the attributes of the training data set and how it was designed. The model was developed with 2100 data of 21 different species of plants infected with fungi disease. The data was collected from the IEEE data repository. The platform provided data for all the 21 plant species used for the development of the data model. From the repository, [4] provided data for Embryophyte plants. [7] provided data for vascular plants. [9] provided data for flowering plants and [8] provided the data for moss plants. These four categories of plants provided 21 species of plants used to develop the data model figure 1.

From figure 1. the attributes and classes of the data set are presented, the data set is made of 21 classes each containing 100 samples of specific plant species infected with fungi. The data is extracted and converted to statistical value using the mathematical activation function before feeding forward for training to generate a reference fungi model.

\section{Model of the artificial neural network}

The system identification process was done using artificial neural network (ANN). The network identifies the output as a nonlinear auto regressive model as shown.

$$
y(k+d)=\boldsymbol{N}(\boldsymbol{y}(\boldsymbol{k}) \cdot \boldsymbol{y}(\boldsymbol{k}-\mathbf{1}) \ldots \boldsymbol{y}(\boldsymbol{k}-\boldsymbol{n}+\mathbf{1}), \boldsymbol{u}(\boldsymbol{k}) \boldsymbol{u}(\boldsymbol{k}-\mathbf{1}) \ldots \boldsymbol{u}(\boldsymbol{k}-\boldsymbol{n}+\mathbf{1}))
$$

Where $\mathrm{u}(\mathrm{k})$ is the sensor output (processed spectral data), $\mathrm{N}$ is the non-linear transfer function of the sensor, $\mathrm{n}$ is the time series of interest for the detection rate, and $\mathrm{y}(\mathrm{k})$ is the ANN output. This output is trained with the training parameters in table 1 using the back-propagation algorithm as shown in the training structure.

$$
\begin{array}{r}
y(k+d)=\boldsymbol{f}(\boldsymbol{y}(\boldsymbol{k}) \cdot \boldsymbol{y}(\boldsymbol{k}-\mathbf{1}) \ldots \boldsymbol{y}(\boldsymbol{k}-\boldsymbol{n}+\mathbf{1}), \boldsymbol{u}(\boldsymbol{k}) \boldsymbol{u}(\boldsymbol{k}-\mathbf{1}) \ldots \boldsymbol{u}(\boldsymbol{k}-\boldsymbol{m}+\mathbf{1})) \\
+\boldsymbol{g}(\boldsymbol{y}(\boldsymbol{k}) \cdot \boldsymbol{y}(\boldsymbol{k}-\mathbf{1}) \ldots \boldsymbol{y}(\boldsymbol{k}-\boldsymbol{n}+\mathbf{1}), \boldsymbol{u}(\boldsymbol{k}-\mathbf{1}) \ldots \boldsymbol{u}(\boldsymbol{k}-\boldsymbol{m}+\mathbf{1})
\end{array}
$$

Where $\mathrm{g}$ is the activation function, $\mathrm{m}$ is the error at each output layer. This model in equation 3 is the training model of the artificial neural network trained using the back propagation algorithm.

Table 1: Neural Network Parameters

\begin{tabular}{|l|l|}
\hline Maximum number of epochs & 100 \\
\hline hidden layers size & 34 \\
\hline Controller training segments & 30 \\
\hline No. delayed reference input & 2 \\
\hline Sensor output & 3 \\
\hline
\end{tabular}




\begin{tabular}{|l|l|}
\hline Sensor plant input & 21 \\
\hline Number of non-hidden layers & 21 \\
\hline Maximum interval per sec & 2 \\
\hline No. delayed controller output & 1 \\
\hline No. delayed sensor output & 3 \\
\hline Minimum value of reference & -0.7 \\
\hline Maximum value of reference & 0.7 \\
\hline
\end{tabular}

The training tool presented in figure 2 showed how the neural network configured the dataset and then train them to generate the fungi model used to improve the sensor. From the tool, the neural network architecture has 21 inputs, which are the various species from the data model. Then the hidden layers were used to activate and train the input data, then predicted the spectral characteristics of the plants.

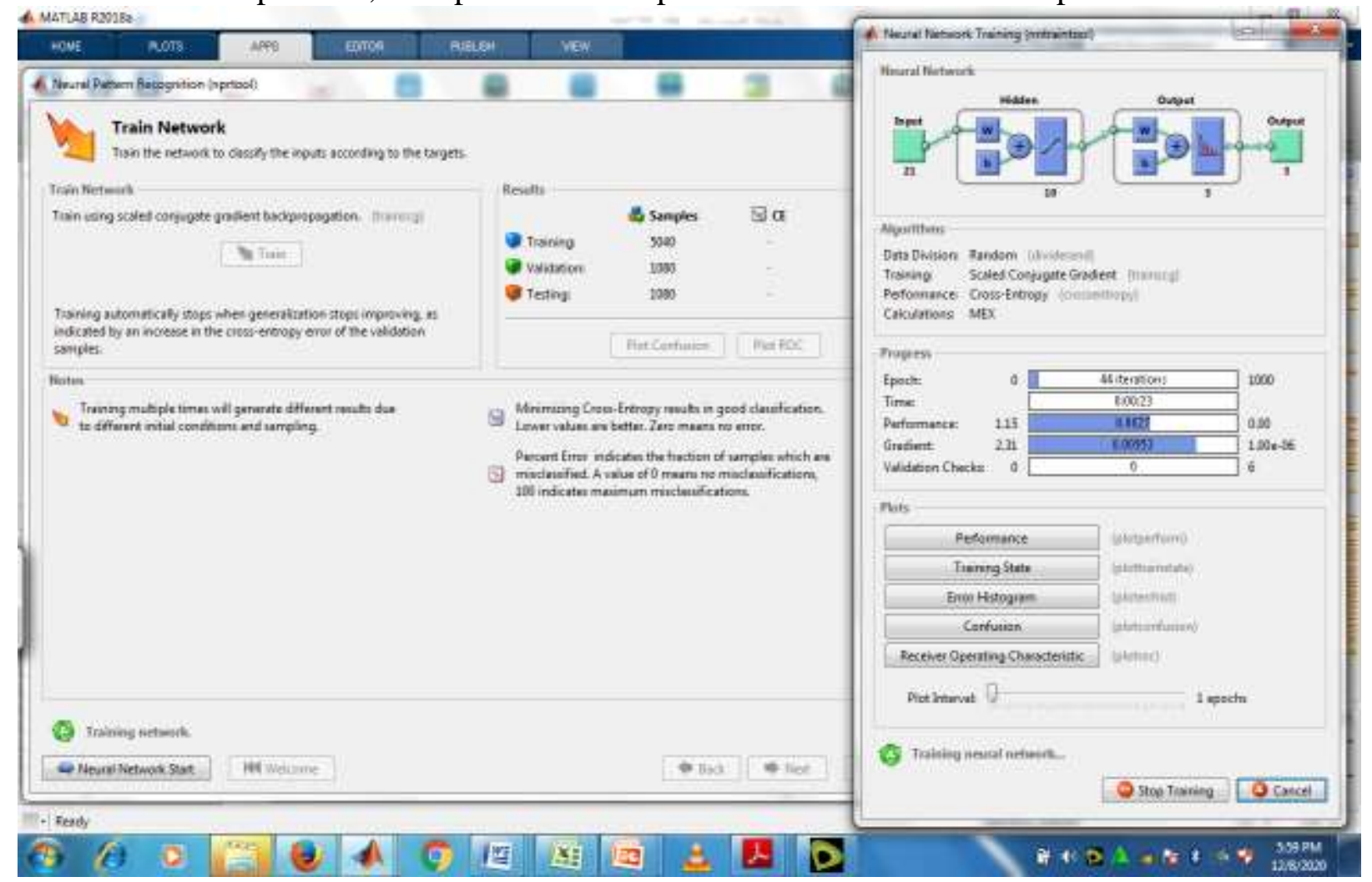

Fig. 2 Neural Network Training Tool

\section{E. Prediction model}

The neural network predicts the network response over the specified input vector (reflectance). The predictor is a numerical optimization program, which determines the feature vectors that minimizes the training performance criterion over the specified horizon as shown below;

\section{Results Discussion}

This section presented the result of the new system developed. The performance was evaluated using the neural network training toolbox to determine the training, test and validation result of the sensor.

\section{Training performance of the ANN}

The neural network training toolbox as shown in the figure 2, intelligently divides the feature vectors extracted from the training dataset in the ratio of 70:15:15 for training, test and validation processes respectively. The aim is to train, self-test and validate the training performance to ensure that the best result is achieved. These results were using tools such as receiver operator characteristics curve (ROC), confusion matrix and training analyzers. The training analyzer is a tool which intelligently monitors the training performance of the sensor using the number of epoch (see table 1) to determine the point at which the best training performance occurs as shown in figure 3. 
The aim here is to achieve a correlated pattern between the test, training and validation graphs, but if otherwise, then the training result is expected to be repeated to achieve a better performance. From the result obtained, it was observed that the best training result was achieved at epoch 9, however, the graph showed that a précised overall training result was not achieved despite the successes. To prove this, the receiver operator curve was called upon as shown in figure 4 to measure the regression value achieved and determine the true training accuracy.
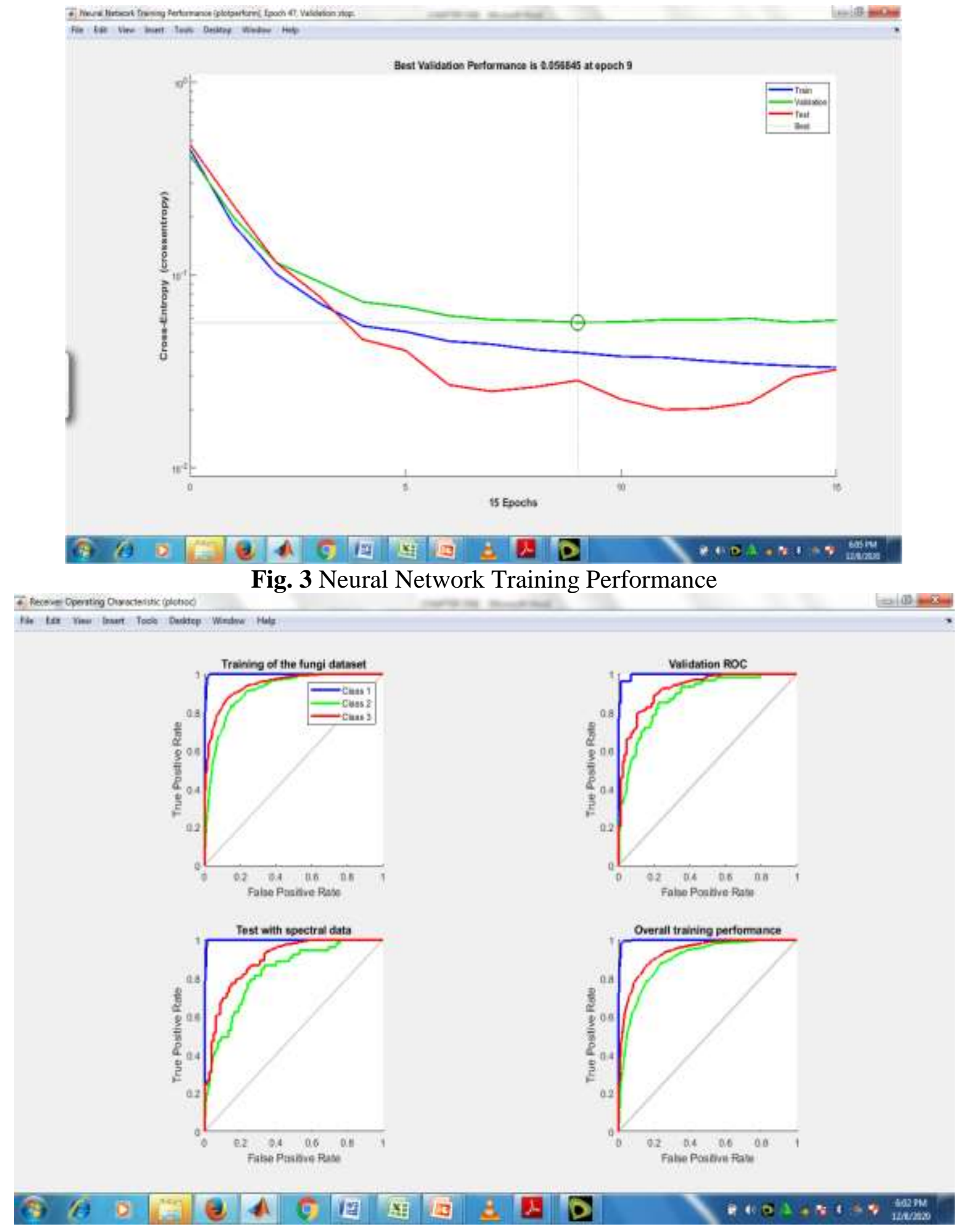

Fig. 4 Regression Analyzer

The result of the regression analyzer in the figure 4 showed the regression performance for the test, train and validation of the sensor. The tool to further evaluate the training of the fungi dataset, the input spectral data that is the test set, validation and their overall average performance, used this measure. 
In the training of the fungi dataset as designed using the model in figure 1 and the backpropagation algorithm, the regression result showed an overall performance of 0.67 . The implication of this result is that the performance of the sensor was good but not satisfactory for a précised fungi detection process. To address this, the training parameters were adjusted increasing the number of hidden layers in the network and then retrained as shown in the training tool in figure 5.

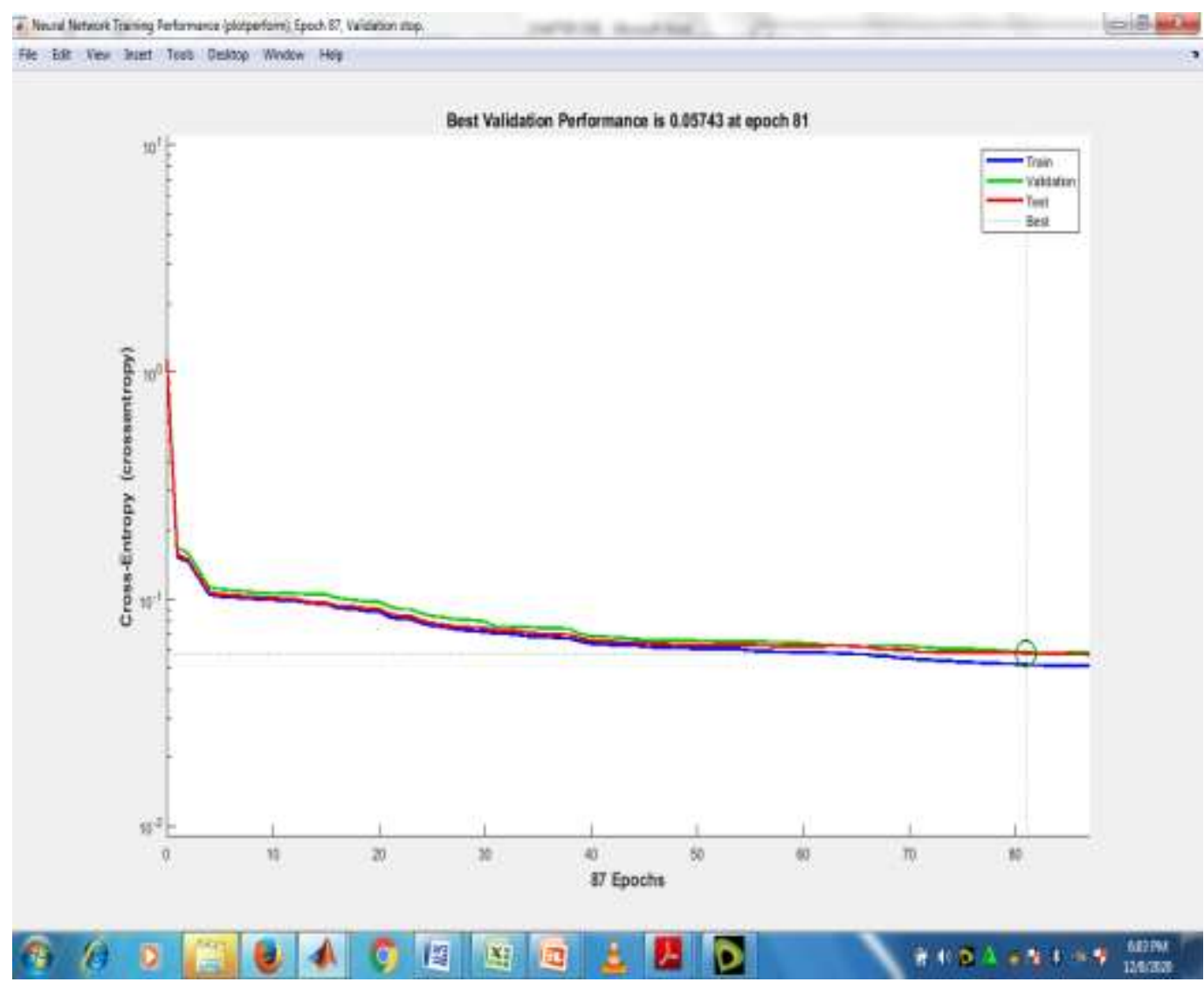

Fig. 5 Neural Network Re-training Performance

IABLE 1 Considerations for the modelling of the $U A V$

\begin{tabular}{|c|c|c|c|}
\hline Parameters & $\begin{array}{l}\text { Pure pursuit } \\
\text { (Empty grid) }\end{array}$ & $\begin{array}{c}\text { Carrot-Chasing } \\
\text { Alapped regive at absolute } \\
\text { beipte) }\end{array}$ & $\begin{array}{c}\text { Carrot-Chaing } \\
\text { (Simple Grid Mlap with } \\
\text { Constraints) }\end{array}$ \\
\hline $\begin{array}{l}\text { Linear } \\
\text { Velocity }\end{array}$ & 0.85 & 09 & 0.7 \\
\hline $\begin{array}{l}\text { Angular } \\
\text { Velocity }\end{array}$ & 2 & 2 & 2 \\
\hline $\begin{array}{c}\text { Look-abead } \\
\text { Distance }\end{array}$ & 095 & 0.99 & 0.8 \\
\hline UAV Radias & 0.5 & 0.7 & 0.8 \\
\hline Gosl Radias & 0.1 & 0.1 & 0.1 \\
\hline $\begin{array}{c}\text { Initial } \\
\text { Orientation }\end{array}$ & 0 & 0 & 0 \\
\hline $\mathrm{X}$ limit & $0-16 \mathrm{~km}$ & $0-25 \mathrm{~km}$ & $0-25 \mathrm{~km}$ \\
\hline Y limit & $0-16 \mathrm{~km}$ & $0-25 \mathrm{~km}$ & $0-25 \mathrm{~km}$ \\
\hline
\end{tabular}

From the result in figure 5, a more correlated relationship between the test, train and validation set was achieved and the best training result was recorded at epoch 81. The implication showed that the classification and learning rate of the sensor using the neural network is very good and will intelligently detect and predict fungi with a high level of accuracy. 
To determine the effectiveness of the training result in a more comprehensive manner, the receiver operator curve in figure 6 was used.

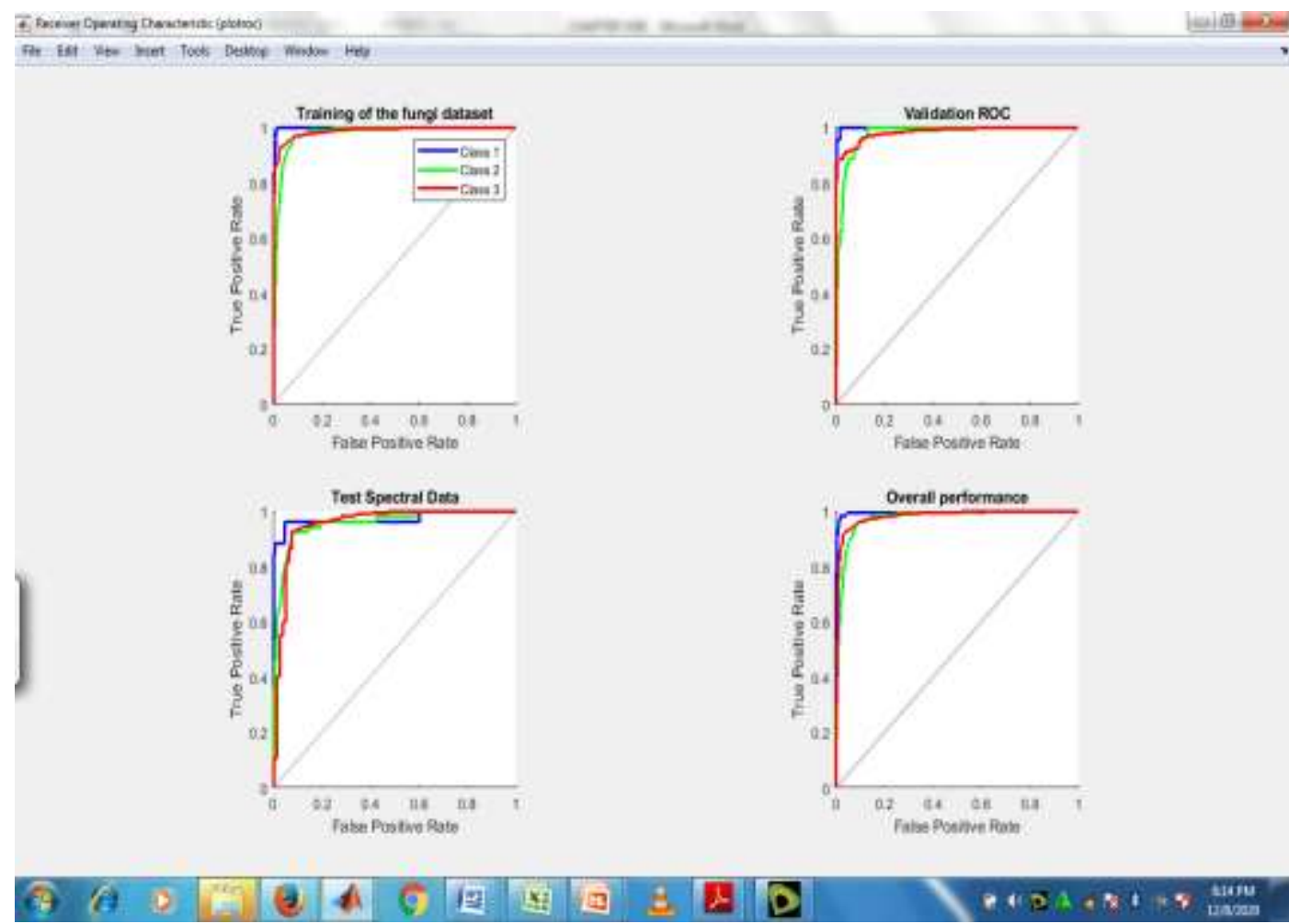

Fig. 6 Receiver Operator Characteristic Curve

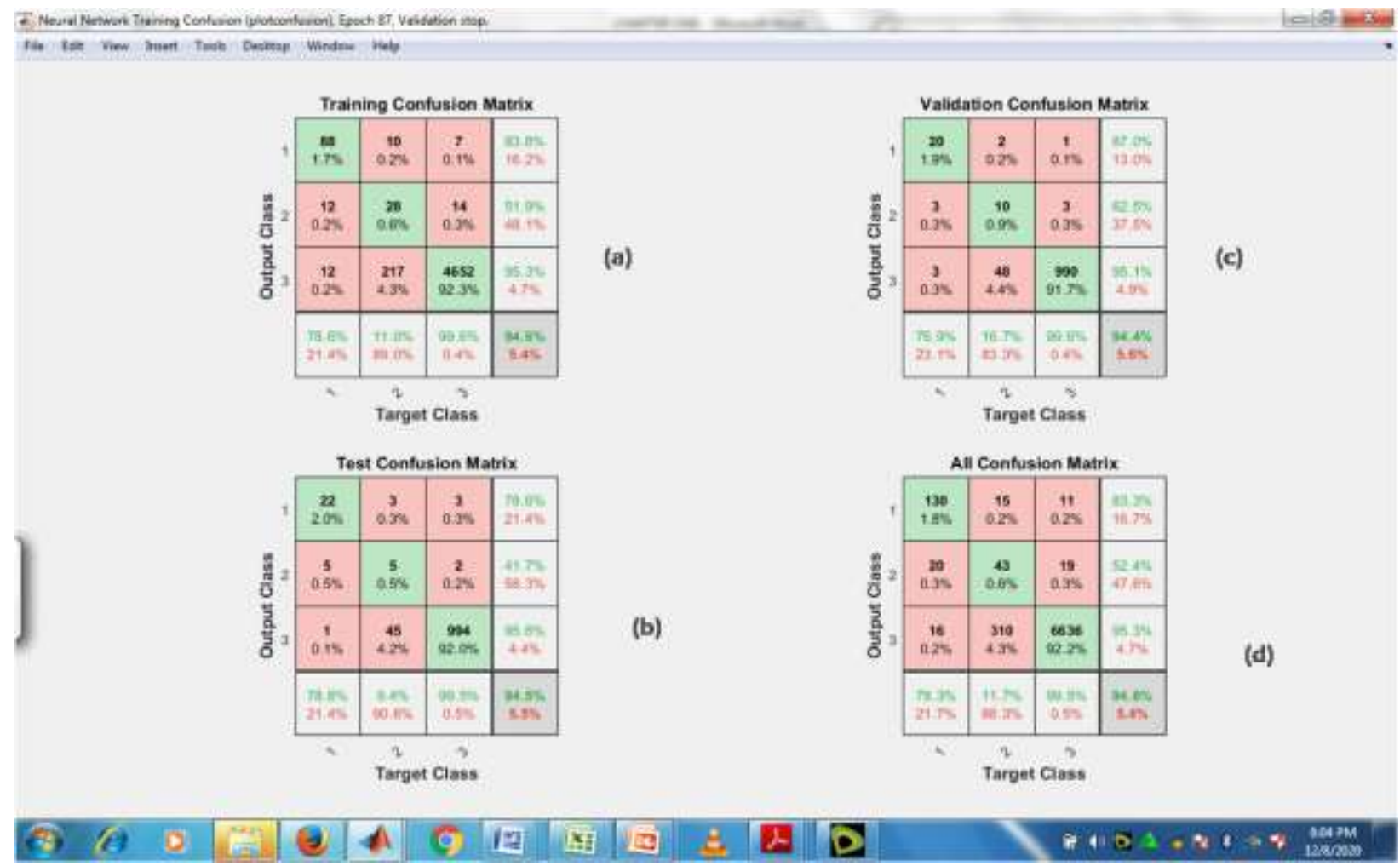

Fig. 7 Result of the Confusion Matrix

The result in figure 7. presented the regression performance of the sensor, showing the test, training, validation and overall performance. From the result, it was observed that the training performance has a true positive rate (TPR) of 0.946 implying that the training was excellent and learned the fungi perfectly. When 
tested with a spectral feature vector the result showed a TPR of 0.945 . The overall performance showed a true positive result of 0.944 inferring that the sensor is reliable with high intelligence in detecting and classifying spectral data from plants.

To measure the accuracy rate of the sensor, the confusion matrix presented in figure 8 was used to show some of the feature vectors extracted from the training dataset and the accuracy of correct and false classification. The training confusion matrix in figure 8 showed the accuracy of correct classification of the fungi training dataset as $94.6 \%$, and when tested with the spectral feature vectors as shown in figure 8 (b), the classification accuracy is $94.5 \%$. This result was validated with an average performance of $94.4 \%$ classification accuracy. The overall performance of the sensor in figure 15(d) based on the rate of correct fungi classification is $94.6 \%$.

\section{Conclusion}

This research has successfully developed an intelligent based smart farm pathogen detection system based on ANN. the disease was revealed as one of the main threats to the success of agricultural practice especially in the African region after an exhaustive literature review of relevant works of literature. The reviews also identified the hyperspectral sensor as the main sensing element in the conventional system but have been limited due to a lack of adaptive intelligence. This was redressed in the study using an artificial neural network and fungi dataset to rectify the problem in the existing system and then develop an intelligent system, for real-time smart farm monitoring. The system developed was tested on a case study farm and the result showed a detection accuracy of $96.61 \%$. It was found that artificial neural networks can be used to improve hyperspectral sensors, a cheap and intelligent fungi sensor was developed.

\section{References}

1. Tanja Folnovic (2019) "Farm Revolution -Sensors for Crop Pest Detection" article on Current and Prospective Methods for Plant Disease Detect

2. Katrin Anne M. (2016) "Plant Disease Detection by Imaging Sensors - Parallels and Specific Demands for Precision Agriculture and Plant Phenotyping" Published Online: 18 Jan 2016 Pages: 241-251;Vol. 100, No. 2.

3. Sen. Heineken Lokpobiri; (2020) "Minister of state for agriculture and rural development, Nigerian; Nigerian food inflation, trending economy.

4. Sharma P, Hans and Gupta S (2020) 'classification of plant leaf disease using machine and image processing techniques' $10^{\text {th }}$ International Conference on cloud computing, data science and engineering, India; pp 480-484.

5. Hetrick, R.L., Rodrigo, O.D. and Bocchini, C.E., 2020. Addressing Pandemic-Intensified Food Insecurity. Pediatrics, 146(4).

6. Pranjali B. Padol, Prof. AnjilA.Yadav, (2016) "SVM Classifier Based Grape Leaf Disease Detection" Conference on Advances in Signal Processing(CAPS) Cummins college of Engineering for Women, Pune.

7. Khirade D and Patil B (2015) 'plant disease detection using image processing' International Conference on computing Communication and Control Automation, India pp 768-771.

8. Panchal P, Raman C and Mantri S (2019) 'plant disease detection and classification using machine learning models' 2019; $4^{\text {th }}$ International Conference on Computational Systems and information Technology for sustainable solution, India pp 1-6.

9. Chapaneri R, Desai M, Ghose S and Das S 'plant disease detection a comprehensive survey' 2020; $3^{\text {rd }}$ International Conference on Communication System Computing and IT applications; India; pp 220-225. 